Gynäkologe 2011 • 44:592-592

DOI 10.1007/s00129-011-2769-8

Online publiziert: 23. Juli 2011

(c) Springer-Verlag 2011

\author{
I. Mylonas · J. Weichert · K. Friese \\ Klinikum Innenstadt - Frauenklinik, LMU München
}

\title{
Virusinfektionen in der Schwangerschaft und im Wochenbett
}

impfung" während der Schwangerschaft, welche mittlerweile auch von der STIKO ausdrücklich empfohlen wird, aufgegriffen und diskutiert. Obwohl wir alle wissen, wie zurückhaltend unsere schwangeren Patientinnen mit dem Thema Impfung umgehen, bleibt die Vakzinierung gegen Influenza eine sehr wichtige - wenn nicht sogar notwendige - prophylaktische Maßnahme zur Vermeidung schwerwiegender maternaler Erkrankungen.

Auch bei einer der wichtigsten geburtshilflichen Infektionen mit einer fetalen Schädigung - einer kongenitalen CMV-Infektion - werden neue diagnostische und vorbeugende Ansätze diskutiert. Weiterhin werden in diesem Heft die interessanten Neuerungen im Geburtsmodus bei einer HIV-Infektion erläutert. Bei den viralen Hepatitiden hat sich ebenfalls in den letzten Jahren einiges verändert. Neben den lang erwarteten S3-Leitlinien werden zurzeit neue therapeutische Optionen (z. B. bei der Hepatitis-B-Infektion) national und international vorgestellt.

Die beiden letzten Themen dieser Ausgabe haben in der klinischen Praxis nichts von ihrer Aktualität verloren. Es scheinen neue Erkenntnisse vorzuliegen, dass bei rezidivierenden Herpes-genitalis-Infektionen eine prophylaktische antivirale Therapie etwa vier bis sechs Wochen vor dem Entbindungstermin die Zahl der Kaiserschnittentbindungen dramatisch senkt. Ebenfalls herrschen immer noch Unklarheiten über das Procedere bei einer während der Schwangerschaft diagnostizierten HPV-Infektion.

Unseren Leserinnen und Lesern wünschen wir eine anregende und lehrreiche
Lektüre dieser infektiologischen Themenausgabe.

Mit herzlichen Grüßen

Ihre

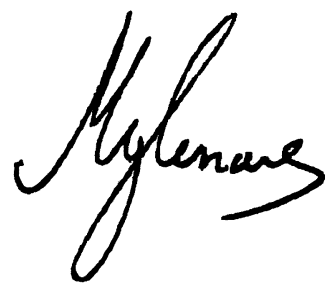

PD Dr. Ioannis Mylonas

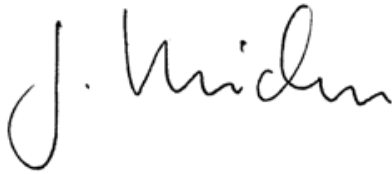

Dr. Jan Weichert

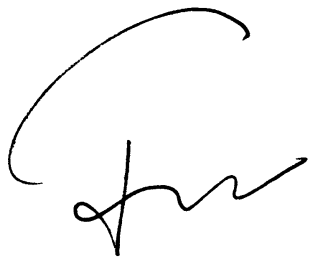

Prof. Dr. Klaus Friese

\section{Korrespondenzadresse}

PD Dr. I. Mylonas

Klinikum Innenstadt - Frauenklinik, LMU München

Maistr. 11, 80337 München

ioannis.mylonas@med.uni-muenchen.de

\section{Literatur}

1. Friese K, Mylonas I (2009) Infektionskrankheiten in Gynäkologie und Geburtshilfe. Gynakologe 42:749-749 